\title{
Direct Evidence for Transplacental Allergen Transfer
}

\author{
ZSOLT SZÉPFALUSI, CHRISTINE LOIBICHLER, JOSEFA PICHLER, KLAUS REISENBERGER, \\ CHRISTOF EBNER, AND RADVAN URBANEK \\ Departments of Pediatrics [Z.S., C.L., J.P., R.U.] and Gynecology and Obstetrics [K.R.], and Institute of \\ General and Experimental Pathology [C.E.], University of Vienna, A-1090 Vienna, Austria
}

\begin{abstract}
Allergies are increasing, and despite deeper insights into the immunologic basis of these diseases, preventive measures are not yet efficient. As the induction of allergic diseases is often triggered in early childhood, perinatal or prenatal preventive strategies would be beneficial. We investigated the transfer of inhalant and nutritive allergens across the human placenta. For this purpose, the maternal side of a placental cotyledon was perfused in vitro with an allergen-containing medium, and a specific ELISA was used to detect the allergens on the fetal side. Both allergens evaluated, birch pollen major allergen Bet v1 and the milk allergen beta-lactoglobulin, could be shown to cross the placenta. The nutritive allergen beta-lactoglobulin was not only transferred across the placenta in all eight experiments, but was also detectable within the first minutes of perfusion. The peak
\end{abstract}

\section{ABSTRACT}

allergen concentration on the fetal side could be increased by addition of human immunoglobulin. For the inhalant allergen Bet $\mathrm{v} 1$, transfer was observed in two of 10 placental experiments, and only if human immunoglobulin was added. A pulsatility wave with a frequency of 30-35 min suggested an active transfer mechanism. We conclude that allergens are actively and selectively transferred across the placenta. Therefore, controlled maternal allergen exposure might offer new ways to induce tolerance to specific allergens in the fetus. (Pediatr Res 48: 404-407, 2000)
Abbreviations
Bet v1, major allergen of Betula verrucosa
BLG, beta-lactoglobulin

There is increasing evidence that the prevalence of allergic diseases is currently rising at an unprecedented rate (1-3). It is also becoming clear that this rise is largely restricted to developed countries, a phenomenon that is widely believed to be linked to a western lifestyle (4). Eliciting agents, preferentially environmental allergens, are predominantly found in indoor environments. Recently, concern has been raised as to any priming effects of exposure to environmental influences even before birth; that is, through the placenta.

Allergen-specific $\mathrm{T}$ cell proliferation in cord blood cells has been demonstrated by many groups and for many different allergens (5-15). Inhalant and nutritive allergens in the form of crude extracts [birch pollen $(5,7)$, house dust mite $(8-10,15)$, timothy grass pollen $(5,7)$, cat fur $(5,7)$, $\operatorname{BLG}(5,7,13,16)$, alpha-casein (13), beta-casein (13), kappa-casein (13), BSA $(5-7,13)$, and ovalbumin $(5,7,11,15,16)]$ and in the form of recombinant allergens [Lol p1 (9), Der p1 (9), Bet v1 (14), and Phl p1 (14)] has been studied. A line of evidence now supports the hypothesis that fetal $\mathrm{T}$ cells are exposed during gestation to

Received July 29, 1999; accepted January 30, 2000.

Correspondence and reprint requests: Zsolt Szépfalusi, MD, Department of Pediatrics, University of Vienna-AKH, Währinger Gürtel 18-20, 1090 Vienna, Austria

Supported by BMWF 49.847, Fonds zur Förderung der wissenschaftlichen Forschung S06701-MED, and Anton Dreher Gedächtnisstiftung 232/93. maternally derived allergens. These allergens may be ingested or inhaled by the mother (14). The time at which maternofetal allergen exposure takes place in the course of a pregnancy is less clear. The current knowledge suggests early times during gestation, presumably around wk 20 of gestation $(5,14)$, Szépfalusi et al. unpublished experiments).

The occurrence of prenatal $\mathrm{T}$ cell priming and its possible impact on later allergic status has been the subject of many rather contradictory papers (5-15). The immunologic mechanisms involved in this process are not clear. They may involve allergen or peptide transfer through the placenta. Alternatively, stimulation of fetal allergen-specific $\mathrm{T}$ cells by anti-idiotypic antibodies is conceivable (17). A recent hypothesis discusses the possibility of a dia-amniotic fluid transfer of maternal IgE antibodies with allergen-presentation through the fetal gutassociated immune system via high-affinity IgE-receptorbearing dendritic cells (18).

The aim of the present study was to investigate the transfer of inhalant and nutritive allergens across the placenta with the help of a placenta model (19-21). For that purpose, after delivery the placenta of healthy mothers was dually perfused. Allergen, diluted in medium, was perfused on the maternal placenta side while the eluate with the transferred allergen was collected on the fetal side. For some experiments, the addition 
of human Ig to the allergen-containing medium was used to study a potential increase of allergen transfer via IgG-mediated mechanisms. BLG as a nutritive allergen and birch pollen major allergen Bet v1 were used because we and others have gained substantial research data with these two allergens, as described in recently published experiments on cord blood $\mathrm{T}$ cells $(5,8,13,14,16)$.

\section{METHODS}

Obstetric history. The obstetric history had been uneventful for the women whose placentas were perfused, and they were taking no drugs. In particular, placentas were only enrolled in the study if there were no signs of maternal infection, no chronic disease necessitating continuous drug use, and no sign of fetal distress. Maternal allergy history was recorded. The kind of anesthesia encompassed analgesic drug medication (in $30 \%$ ), epidural anesthesia $(40 \%)$, and no medication $(30 \%)$. All placentas used were from term deliveries with a placental weight of 500 to $800 \mathrm{~g}$.

Placentas. Placentas from uncomplicated term pregnancies were obtained after vaginal delivery or cesarean section. Placental transfer of the allergens BLG and Bet v1 was investigated with a double-sided open ex vivo perfusion model (1923 ). For an experiment to be included in the analysis, arterial inflow had to equal venous outflow. Evans blue, a dye not capable of crossing the placental barrier, was added to the maternal circuit to macroscopically document the integrity of the membranes at the beginning and the end of each experiment, thereby excluding gross leakage. Perfusion with the allergen-containing medium was continued throughout the experimental period. Studies on placentas after delivery have been approved by the institutional review board.

Medium. The perfusion medium contained $11 \mathrm{~g}$ of technetium-Medium 199 (Biochrom KG, Berlin, Germany), $3 \mathrm{~g}$ of bovine albumin (Behringwerk AG, Marburg, Germany), $20 \mathrm{~g}$ of dextran-T 40 (Pharmacia, Uppsala, Sweden), $10 \mathrm{~mL}$ of sodium hydrogen carbonate $8.4 \%$, and $1000 \mathrm{~mL}$ of distilled water. To achieve oxygen saturation, the solution was aerated with $95 \%$ oxygen and $5 \%$ carbon dioxide for at least $30 \mathrm{~min}$ in a water bath at $37^{\circ} \mathrm{C}$. The $\mathrm{pH}$ was adjusted by addition of bicarbonate and $1 \mathrm{M} \mathrm{HCl}$ to $\mathrm{pH}$ 7.4.

Allergens. A nutritive and an inhalant allergen of comparable molecular weight were chosen. Birch pollen major allergen Bet v1 (Betula veruccosa, MW $17 \mathrm{kD}$ ) was added at a concentration of $0.2 \mu \mathrm{g} / \mathrm{mL}$, and cow's milk whey protein BLG (MW $18.5 \mathrm{kD}$ ) was used at a concentration of $2.0 \mathrm{mg} / \mathrm{mL}$. The allergen concentrations were deduced from very few publications in animal models. In the first $10 \mathrm{~min}$, samples of fetal perfusate were collected every minute, and thereafter in intervals of $5 \mathrm{~min}$. In some experiments, human Ig (Octagam, Octapharma, Vienna, Austria) as a source of polyclonal antibodies was added to the perfusate at a final concentration of $10 \%$.

Allergen determination by ELISA. The concentrations of BLG and Bet v1 were measured by ELISA techniques. Both assays were established or adjusted in our laboratory. For the detection of Bet v1, the following antibodies were used: mono- clonal mouse anti-Bet v1 (BIP 1), polyclonal rabbit anti-Bet v1, and swine anti-rabbit alkaline phosphatase (Sigma Chemical Co., St. Louis, MO, U.S.A.). For the detection of BLG, the following antibodies were used: rabbit-anti-BLG (affinity purified) and rabbit anti-BLG alkaline phosphatase (Bethyl, Montgomery, AL, U.S.A.). The assays for BLG and Bet v1 had a sensitivity of $5 \mathrm{ng} / \mathrm{mL}$ and $0.2 \mathrm{ng} / \mathrm{mL}$, respectively.

\section{RESULTS}

General. Eighteen preparations fulfilled the perfusate recovery criteria. 10 of the 18 were used to study maternal to fetal Bet v1 clearance, and eight were used to measure maternal to fetal BLG clearance. All placentas were term and from otherwise healthy mothers delivering at the General Hospital of Vienna. The allergic history was registered.

BLG crosses the placenta in all experiments. The eluates of the eight placental perfusions with BLG $(500 \mathrm{mg} / 250 \mathrm{~mL})$ were analyzed with a sandwich ELISA using BLG-specific antibodies in capture and detecting position. In all eight placenta experiments, BLG was detected at the fetal side. Furthermore, BLG appeared within the first minutes as shown by its presence in the first eluate sample (Fig. 1A). The initial concentration varied between 50 and $250 \mathrm{ng} / \mathrm{mL}$. A steady increase of the BLG concentration was detected during the observation period of $120 \mathrm{~min}$ (Fig. 1A). Adding human serum (in the form of pooled human serum prepared for i.v. Ig therapy) to the perfusion medium resulted in a slightly higher initial BLG concentration in the first eluate with no additional increase during the observation period (Fig. 1B). As shown in Figure 1, all eluate curves show pulsatility peaks in 30- to 35-min intervals. Pulsatility peaks have been described for the release of many hormones that are under hypothalamic control. Although the pulsatility peaks in hormone release are more pronounced, this observation may underline an active transport mechanism operative in diaplacental allergen transfer.

Bet 1 crosses the placenta occasionally and independently of human Ig. The eluates of the 10 placental perfusions with Bet v1 $(50 \mu \mathrm{g} / 250 \mathrm{~mL})$ were analyzed with a sandwich ELISA using Bet v1-specific monoclonal and polyclonal antibodies at capture and detecting positions, respectively. Of 10 placenta experiments, allergen transfer of Bet v1 was observed in two cases (Fig. 2). In contrast to the transfusion experiments using BLG, Bet v1 was only detectable on the fetal side if human serum was added to the perfusion medium. This observation may enforce the notion of a transport mechanism with involvement of Ig receptors (transcellular transport, paracellular transport). Neonatal Fc-receptor has been shown to be present and involved in such transplacental mechanisms, and it could be suggested that this mechanism is also operative in the observed condition.

\section{DISCUSSION}

This study is the first to directly investigate allergen transfer across the human placenta. For this purpose, an ex vivo placenta model was used. The data show that allergen present in the maternal blood circulation (as present in the perfusion medium) is readily detected in the fetal blood circulation, 




B

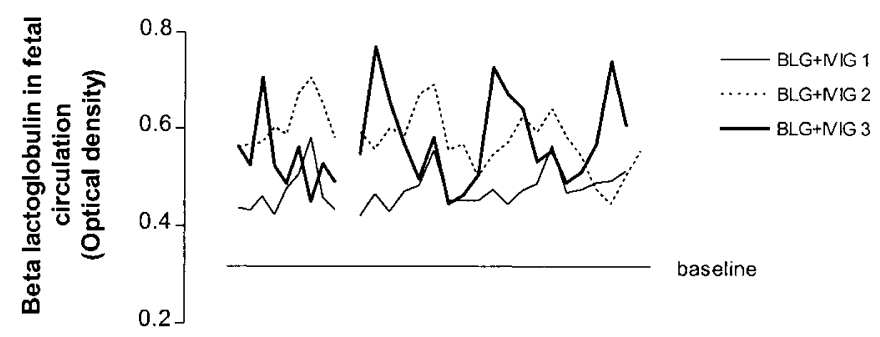

$\begin{array}{lllllllll}02 & 4 & 6 & 8 & 10 & 30 & 1 & 1 & 1 \\ 0 & 10 & 90 & 110\end{array}$

Placenta perfusion period $(\mathrm{min})$

Figure 1. $A$, transplacental transfer of nutritive allergen (BLG) occurs universally. Placental cotyledons $(n=8)$ were perfused with BLG-supplemented medium, and allergen transfer was detected by a BLG-specific ELISA. Three representative experiments are shown (BLG 1,2, and 3). Perfusion experiments were performed for $120 \mathrm{~min}$, and allergen detection was expressed as OD, with 0.3 OD defined as background level of the assay. $B$, transplacental transfer of BLG is enhanced by addition of human IgG and shows a pulsatility wave curve. Placental cotyledons $(n=3)$ were perfused with BLGsupplemented medium and human serum (Octagam, Actapharma, Vienna), and allergen transfer was detected by a BLG-specific ELISA. Three experiments are shown (BLG + IVIG 1, 2, and 3). Perfusion experiments were performed for $120 \mathrm{~min}$, and allergen detection was expressed as OD, with $0.3 \mathrm{OD}$ defined as background level of the assay.

providing direct evidence for transplacental maternofetal allergen transfer. This finding was true for both allergens investigated, which differed only in terms of permeability kinetics and allergen concentrations used.

The incidence of allergic disease has increased during the past $25 \mathrm{y}$, not simply as a result of increased awareness (1-3). Beside a genetic propensity and the influence of environmental allergens in countries with a western lifestyle, research has focused on basic mechanisms governing allergic reaction. For a disease beginning mostly early in infancy, it appears necessary to first understand the cellular and molecular mechanisms underlying the induction and expression of allergic reactions (24). A line of evidence now supports the hypothesis that fetal $\mathrm{T}$ cells are exposed during gestation to maternally derived allergens. These allergens could be ingested or inhaled by the mother (14). The time and way at which this allergen exposure takes place in the course of a pregnancy is less clear. The current knowledge suggests early time points during gestation, presumably around wk 20 of gestation $(5,14)$, Szépfalusi et al. unpublished experiments). Although transplacental passage of allergens appears possible, the role of the placenta in the

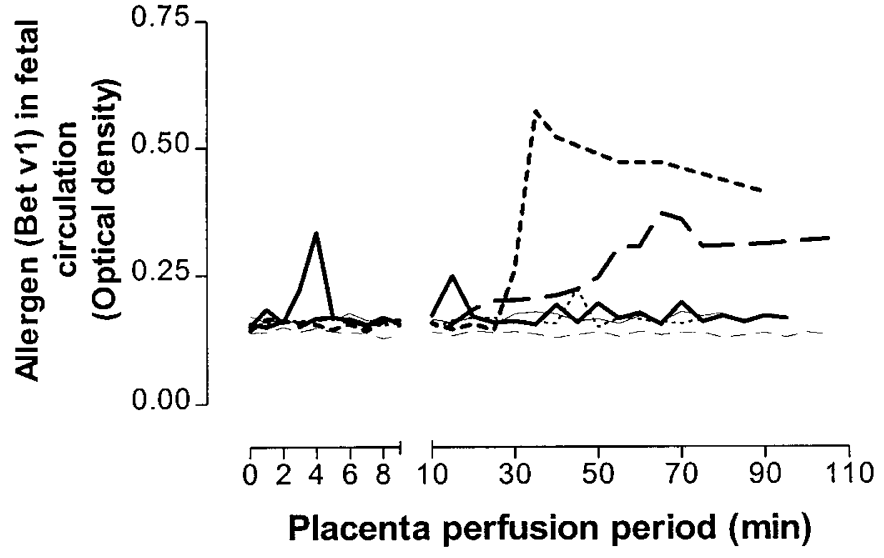

Figure 2. Transplacental transfer of inhalant seasonal allergen (Bet v1) reflects a rare phenomenon. Placental cotyledons $(n=10)$ were perfused with Bet v1-supplemented medium, and allergen transfer was detected by a Bet v1-specific ELISA. Six representative experiments are shown. Perfusion experiments were performed for $120 \mathrm{~min}$, and allergen detection was expressed as $\mathrm{OD}$, with $0.2 \mathrm{OD}$ defined as background level of the assay.

permeability of particular allergens has not been investigated yet in humans (5-15). In rat, Dahl et al. (25) have shown that chicken ovalbumin and cow milk BLG fed orally to rats could be readily detected in fetal blood. After a feed of $100 \mathrm{mg}$ of antigen, the antigen concentration in the maternal blood circulation was $10-20 \mu \mathrm{g} / \mathrm{mL}$, and the corresponding concentration in the fetal blood circulation for the same antigen was 1-2 $\mu \mathrm{g} / \mathrm{mL}$. In our experiments, the ratio of allergen in the perfusate (maternal circulation) and of allergen in the eluate (fetal circulation) was 1000:1, illustrating that most of the allergen is not transferred across the placenta. It is debatable whether $50-250 \mathrm{ng} / \mathrm{mL}$ of allergen detected in the eluate is sufficient to induce an immune response, but it appears possible keeping in mind the data of allergen-specific lymphoproliferation of cord blood cells (5-15). These papers have shown in common that priming of fetal lymphocytes occurs ubiquitously irrespective of a particular allergen dose. In human placenta investigations, it has been shown previously that alpha-fetoprotein readily crosses the placenta barrier, with a net permeability increase from the fetal to maternal circulation (23). As the permeability of alpha-fetoprotein was proportional to the coefficient of free diffusion in water, a transfer by paracellular diffusion was postulated.

In our experiments, we investigated two different allergens of similar molecular weight and similar coefficient of free diffusion in water: the perennial nutritive allergen BLG and the seasonal inhalant Bet v1. This choice has been deduced from recently published data on cord blood mononuclear cell reactivity to these two allergens. Hence, we and others could observe an ubiquitous cord blood cellular reactivity to BLG (5, $8,13,16)$, whereas only occasional and in these cases maternal allergen exposure-dependent reactivity was detectable for Bet v1 (14). To explain these observations some hypotheses were discussed. The perennial and therefore constant presence of nutritive allergens in the maternal blood circulation throughout gestation, e.g. BLG, may lead to constant transfer of BLG in minute amounts and lead to priming of the fetal immune system. On the other hand, for the seasonal allergen Bet v1 the 
situation is more restricted because the allergen exposure period is short (depending on the birch pollen season). Here, only those mothers exposed to the allergen could also transfer the allergen via the placental barrier. Unfortunately, the use of other inhalant and nutritive perennial allergens (house dust mite allergen, ovalbumin) show that this hypothesis is not operational universally. Although house dust mite allergen is thought to be perennially present in the indoor environment, published (9) and unpublished data (Kopp et al.; Herz, personal communications) suggest that only a limited number of allergen-exposed mothers transfer the allergen to the fetus leading to a detectable $\mathrm{T}$ cell priming effect. In addition, with the nutritive perennial allergen ovalbumin, only occasional cellular reactivity was detectable in cord blood cells. Therefore, the simple presence of allergen in the maternal blood circulation is per se not sufficient to explain the different lymphoproliferative reactivity rates in cord blood cells. To explain this, we propose that the placental barrier plays an active role in the decision of whether an antigen crosses the placenta or not. In that way, the transplacental transfer of allergens may be part of a mechanism protecting the fetus. Before birth, professional antigenpresenting cells in the peripheral blood (dendritic cells) are not equipped with a mature set of costimulatory molecules enabling them to present professionally antigen to circulating antigen-specific T cells. Moreover, such stimulation of T cells would lead to tolerance induction to those antigens encountered before birth. It is therefore tempting to speculate that controlled maternal allergen exposure could actively induce allergen-specific tolerance in the fetus. In fact, allergen avoidance protocols by pregnant mothers have in the past not been shown to be beneficial in avoiding subsequent development of allergic disease in the child. This hypothesis is proposed as an alternative pathway to the transamniotic transfer of maternal $\operatorname{IgE}$ and potentially also allergens encountered by the mother, which through gut-associated IgE receptor-mediated antigen focusing would lead to induction of allergic sensitization (18). In summary, both mechanisms, a transplacental allergen transfer and a transamniotic IgE and allergen transfer, could be operative together, and the balance between both systems would skew toward tolerance induction or toward specific allergic sensitization.

We thank Prof. Husslein (Vienna, Austria) for his help with this study.

\section{REFERENCES}

1. Aberg N, Hesselmar B, Aberg A, Eriksson B 1995 Increase of asthma, allergic rhinitis and eczema in Swedish schoolchildren between 1979 and 1991. Clin Exp Allergy $25: 815-819$
2. Björksten B, Suoniemi I, Koski V 1980 Neonatal birch pollen contact and subsequent allergy to birch pollen. Clin Allergy 10:585-590

3. Shaheen S 1995 Changing patterns of childhood infection and the rise of allergic disease. Clin Exp Allergy 25:1034-1037

4. Wichmann H 1996 Possible explanation for the different trends of asthma and allergy in East and West Germany. Clin Exp Allergy 25:1034-1037

5. Jones A, Miles E, Warner J, Colwell B, Bryant T, Warner J 1996 Fetal peripheral blood mononuclear cell proliferative responses to mitogenic and allergenic stimuli during gestation. Pediatr Allergy Immunol 7:109-116

6. Kondo N, Kobayashi Y, Shinoda S, Kasahara K, Kameyama T, Iwasa S, Orii T 1992 Cord blood lymphocyte responses to food antigens for the prediction of allergic disorders. Arch Dis Child 67:1003-1007

7. Miles EA, Jones AC, Warner JA, Warner JO 1995 Lymphocyte proliferative responses to allergens and mitogens during the first year in babies born to atopic parents. J Allergy Clin Immunol 95:381

8. Miles E, Warner J, Jones A, Colwell B, Bryant T, Warner J 1996 Peripheral blood mononuclear cell proliferative responses in the first year of life in babies born to allergic parents. Clin Exp Allergy 26:780-788

9. Picinni M-P, Mecacci F, Sampognaro S, Manetti R, Parronchi P, Maggi E, Romagnani S 1993 Aeroallergen sensitization can occur during foetal life. Int Arch Allergy Immunol 102:301-303

10. Piastra M, Stabile A, Fioravanti G, Castanola M, Pani G, Ria F 1994 Cord blood mononuclear cell responsiveness to beta-lactoglobulin: $\mathrm{T}$ cell activity in "atopyprone" and "non-atopy-prone" newborns. Int Arch Allergy Immunol 104:358-365

11. Prescott SL, Macaubas C, Yabuhara A, Venaile T, Habre W, Loh R, Sly PD, Holt PG 1997 Developing patterns of T cell memory to environmental allergens in the first two years of life. Int Arch Allergy Immunol 113:75-79

12. Prescott SL, Macaubas C, Holt BJ, Smallacombe TB, Loh R, Sly PD, Holt PG 1998 Transplacental priming of the human immune system to environmental allergens: universal skewing of initial $\mathrm{T}$ cell responses toward the $\mathrm{TH} 2$ cytokine profile. J Immunol 160:4730-4737

13. Szépfalusi Z, Nentwich I, Gerstmayr M, Jost E, Todoran L, Gratzl R, Herkner K, Urbanek R 1997 Prenatal allergen contact with milk proteins. Clin Exper Allergy 27:28-35

14. Van Duren-Schmidt K, Pichler J, Ebner C, Förster E, Bartmann K, Urbanek R, Szépfalusi Z 1997 Prenatal allergen contact with aeroallergens. Pediatr Res 41:1-4

15. Yabuhara A, Macaubas C, Prescott S, Venaile T, Holt B, Habre W, Sly P, Holt P 1997 TH2-polarized immunological memory to inhalant allergens in atopics is established during infancy and early childhood. Clin Exp Allergy 27:1261-1269

16. Warner JA, Miles EA, Jones AC, Quint DJ, Colwell BM, Warner JO 1994 Is deficiency of interferon gamma production by allergen triggered cord blood cells a predictor of atopic eczema? Clin Exp Allergy 24:423-430

17. Hahn-Zoric M, Carlasson B, Björkander J, Osterhaus A, Mellander L, Hanson L 1992 Presence of non-maternal antibodies in newborns of mothers with antibodydeficiency. Pediatr Res 32:150-154

18. Jones CA, Warner JA, Warner JO 1999 Fetal swallowing of IgE. Lancet 351:1859

19. Fortunato S, Bawdon R, Swan K 1992 Transfer of Timentin across the in vitro perfused human placenta: comparison with other agents. Am J Obstet Gynecol 167:1595-1599

20. Schneider H, Panigel M, Dancis J 1972 Transfer across the perfused placenta of antipyrine, sodium and leucine. Am J Obstet Gynecol 114:822-828

21. Schneider H, Huch A 1985 Dual in vitro perfusion of an isolated lobe of human placenta: method and instrumentarium. Contrib Gynecol Obstet 13:40-47

22. Reisenberger K, Egarter C, Sternberger B, Eckenberger P, Eberle E, Weissenbacher E 1996 Placental passage of angiotensin-converting enzyme inhibitors. Am J Obstet Gynecol 174:1450-1455

23. Brownbill P, Edwards D, Jones C, Mahendran D, Owen D, Sibley C, Johnson R, Swanson P, Nelson D 1995 Mechanisms of alpha-fetoprotein transfer in the perfused human placenta cotyledon from uncomplicated pregnancy. J Clin Invest 96:2220 2226

24. Holt P 1998 Programming for responsiveness to environmental antigens that trigger allergic respiratory disease in adulthood is initiated during the perinatal period. Environ Health Perspect 106:795-800

25. Dahl G, Telemo E, Weström B, Jakobsson I, Lindberg T, Karlsson B 1984 The passage of orally fed proteins from mother to foetus in the rat. Comp Biochem Physiol 77A:199-201 